EPJ Web of Conferences 49, 11001 (2013)

DOI: $10.1051 /$ epjconf/20134911001

C) Owned by the authors, published by EDP Sciences, 2013

\title{
LHCf and connection to high energy cosmic rays
}

\author{
Yoshitaka Itow ${ }^{1,2, a}$ for the LHCf collaboration. \\ ${ }^{1}$ Solar-Terrestrial Environment Laboratory, Nagoya University \\ ${ }^{2}$ Kobayashi Maskawa Institute for the Origin of particles and the Universe.
}

\begin{abstract}
An extensive air shower is only the method to observe ultra high energy cosmic rays. Implications of air showers always rely on hadron interactions at such high energy. Hadron collider data can give an unique opportunity to verify and to improve knowledge on hadron interactions relevant for air showers. The LHCf experiment provides data for particle productions at very forward region in LHC to verify the forward energy spectra from the collisions. Recent results of LHCf and its connection to air shower experiments are overviewd.
\end{abstract}

\section{Introduction}

Existence of ultra high energy cosmic rays of which energy reach $10^{20} \mathrm{eV}$ is a very interesting and also enigmatic topic. Their origins, composition and propagation mechanism are yet not fully known. If UHECR protons with more than several $10^{19} \mathrm{eV}$ travel from the source at a few hundred Mpc distance, they interact with $\mathrm{CMB}$ photons via $\Delta$ resonance and should be attenuated. This so-called GZK cut-off feature would be an important evidence that such high energy proton travel through $\sim 100$ Mpc distance. Recently two air shower experiments, Pierre-Auger Observatory (PAO) and Telescope Array (TA) have been carried using air fluorescence telescopes as well as ground detector arrays with huge coverage. They revealed existence of cut-off feature of energy spectra consistent to GZK [1] [2]. However their chemical composition measurements, i.e. proton primary or heavy ion primary, are not consistent with each other [1] [2]. Experimental situation is still controversial.

Chemical composition of high energy cosmic rays is often statistically determined by a distribution of shower max height of air showers $\left(X_{\max }\right)$. For primary cosmic rays of energy $E$, energy per nucleon for heavy ion primary $E / A$ is smaller than that of proton primary. Thus $X_{\text {max }}$ 's for proton primary showers are likely larger and more fluctuated than those for heavy primary. By using telescopes, longitudinal profile of air shower can be observed by imaging of air fluorescence lights produced from electro-magnetic component of air showers.

However air shower development is not only correlated with primary compositions but also largely depends on high energy hadron interactions for following points; 1) inelastic cross sections, 2) forward energy spectra of secondary particles, 3) inelasticity of collisions, and so on.

\footnotetext{
ae-mail: 190 itow@stelab.nagoya-u.ac.jp
}

Some of these parameters, for example inelastic cross section $\sigma_{\text {inel }}$ have been already checked by collider experiments. For example $\sqrt{s}=14 T e V$ pp collisions correspond to interactions of cosmic rays with $10^{17} \mathrm{eV}$ which is just a few order smaller than the GZK energy regions. Cosmic rays in the LHC collision energy region is also covered by the existing KASCADE-GRANDE [3] air shower detector and also will be covered by low energy extension plans of PAO and TA, i.e. HEAT [4] and TALE [5], respectively. Thus $10^{17} \mathrm{eV}$ is interesting energy where both air showers and LHC minimum bias data can be directly compared.

Recently LHC data have been tested with many versions of PYTHIA as well as various cosmic ray interaction models which are commonly used in cosmic ray air shower experiments such as SYBILL [6], QGSJET II [7], DPMJET3 [8], and EPOS [9]. These cosmic ray interaction models more or less consider multi-pomeron interactions in the Gribov-Regge type theory. It has been found the cosmic ray interaction models show better agreement in minimum bias events observed at LHC, comparing the existing PYTHIA-based models [10] [11] [12]. Especially forward interactions have not been precisely studied, where non-perturbative QCD plays an essential role. This forward region is the most relevant to air shower development, because most of the collision energy concentrate in the forward as shown in Fig. 1. Since most of the central detectors of the LHC experiments only cover the region $\eta<4$, dedicated detector is needed to cover the much larger $\eta$ region. There are unique forward particle detectors, TOTEM T1/T2 [13] or CMS CASTOR [12], in LHC. Among them LHCf has unique good capability of electro-magnetic calorimetry, impact parameter measurement, electro-magnetic/hadron separation, and invariant mass reconstruction. In air shower, more than $90 \%$ of primary energy is converted to electro-magnetic component. Thus it is essentially important to measure the 

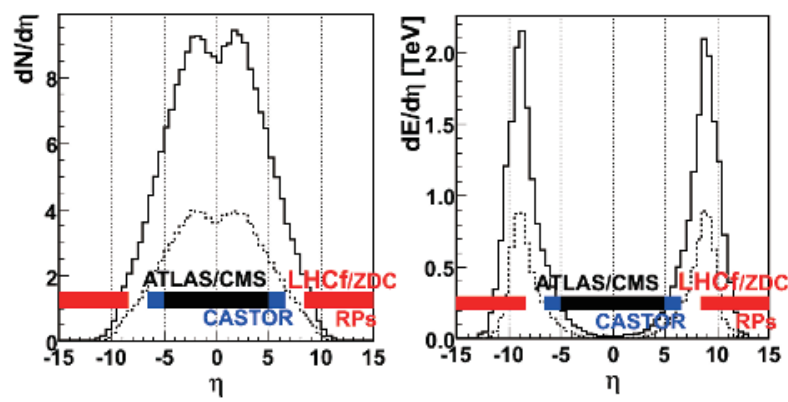

Figure 1. The multiplicity (left) and the energy flow (right) of secondary particles as a function pseudorapidity at $7 \mathrm{TeV} p-p$ collisions. Also shown typical pseudorapidity coverage of existing LHC detectors.
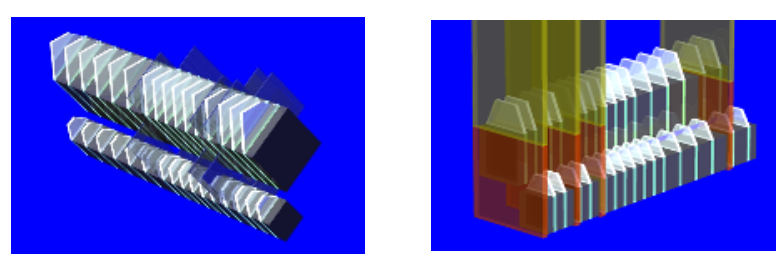

Figure 2. The LHCf detector Arm1(left) and Arm2(right).

energy spectra of electro-magnetic component produced by secondary particles at very forward. The LHCf experiment is dedicated for this purpose and aiming to reduce uncertainty in shower development [14]. In this article, the LHCf experiment and current results will be presented.

\section{LHCf experiment}

The LHCf experiment has two independent detectors, Arm1 and Arm2, installed in the $10 \mathrm{~cm}$ wide slot in TAN $140 \mathrm{~m}$ apart from IP1. Here is a Y-shaped beam chamber downstream the D1 magnet which sweeps away all the charged particles. Therefore only neutral particles emitted at "0 degree" of the interaction region can be detected. This location covers the pseudo-rapidity from $\eta=8.6$ to infinity. Schematic view of the detector is shown in Fig. 2. Each detector consisted of a small and a large sampling calorimeters with 16 layers of plastic scintillators interleaved with tungsten of 2 radiation lengths. The dimensions of calorimeters in Arm1 (Arm2) were $2 \mathrm{~cm} \times 2 \mathrm{~cm}$ ( $2.5 \mathrm{~cm} \times 2.5 \mathrm{~cm})$ and $4 \mathrm{~cm} \times 4 \mathrm{~cm}(3.5 \mathrm{~cm} \times 3.5 \mathrm{~cm})$ for small and large calorimeters, respectively. Total radiation length and interaction lengths were $44 \mathrm{X}_{0}$ and $1.6 \lambda$, respectively. Each detector had 4 layers of position sensitive layers using $1 \mathrm{~mm}$ plastic scintillating fiber (Arm1) or Si strip detectors (Arm2). These layers provided shower incident position with accuracy of $1 \mathrm{~mm}$ and $200 \mu \mathrm{m}$ for scintillating fibers and Si detector, respectively. The incident position information was used to correct position dependent shower leakage effects. Also they were used to discriminate multiincident events. After these corrections typical energy resolution and position resolution for Arm1 (Arm2) were 5\%
(5\%) and $170 \mu \mathrm{m}(40 \mu \mathrm{m})$, respectively. Trigger threshold was about $100 \mathrm{GeV}$ as gamma-ray energy for each tower. In addition, a Front Counter (FC) made of plastic scintillators was inserted in front of each calorimeter to provide luminosity normalisation, which was calibrated during the operations for Van der Meer scan [15]. All detail information can be found elsewhere [16] [17] [18].

Figure 3 shows the scatter plot for the energy vs $p_{T}$ of gamma rays from $7 \mathrm{TeV} p$ - $p$ collisions. Here typical $p_{T}$ of secondary particles is about $0.4 \mathrm{GeV} / c$, which has been commonly known in air shower phenomena. The lines corresponds to pseudorapidity values $\eta=8.77,8.40$, 7.60, 6.91 and 5.99 (from bottom to top), respectively. The $\eta$ acceptance of LHCf is roughly $\eta>8.77$, corresponding to the acceptance of $p_{T}$ less than several hundreds $\mathrm{MeV} / c$. Main contribution to the particle production in this $P_{T}$ region is considered to be projectile fragmentation. LHCf observes collisions and particle productions essentially in non-perturbative QCD regime.

In 2010, data taking at $\sqrt{s}=7 \mathrm{TeV}$ and $0.9 \mathrm{TeV}$ had been completed. Data taking was carried out in the machine commissioning phase with luminosity less than $\sim 10^{30} \mathrm{~cm}^{2} \mathrm{~s}^{-1}$. Before high luminosity $\left(>10^{31} \mathrm{~cm}^{2} \mathrm{~s}^{-1}\right)$ operation at LHC started in July 2010, the detectors were de-installed from the TAN. One of the detector Arm2 had been installed again in the tunnel for $\mathrm{p}-\mathrm{Pb}$ collisions in 2012 winter. After successful data taking of $\mathrm{p}-\mathrm{Pb}$ collisions, the detector has been removed again for LSS1.

\section{Energy spectra for the single $\gamma$ sample}

Obtained data was analysed to estimate shower energy by sum of scintillator layer signals, which were corrected for shower leakage effect by the incident position reconstructed from position sensitive layers. Energy scale was calibrated by a $100-200 \mathrm{GeV}$ electron beams at SPS-H4 and also checked by invariant masses of reconstructed $\pi^{0}$. Here single-incident events were selected by the position sensitive layers. Generally electro-magnetic cascade showers reach shower maximum at around several radiation lengths, while hadronic showers deposit their energies almost randomly. We defined "L90\%" which is the radiation length where $90 \%$ of visible shower energy is contained. Finally electro-magnetic showers were selected by using L90\%.

Figure 4 shows the measured energy spectra of gamma rays produced in the pseudorapidity regions $8.81<|\eta|<$ 8.99 and $|\eta|>10.94$ at $\sqrt{s}=7 \mathrm{TeV} p$ - $p$ collisions [19]. Also shown in Fig. 5 the energy spectra of gamma rays produced in the pseudorapidity regions $8.77<|\eta|<9.46$ and $|\eta|>10.15$ at $\sqrt{s}=0.9 \mathrm{TeV} p$ - $p$ collisions [20]. Here the measured gamma ray energy spectra were compared with the MC prediction by various interaction models, SYBILL, DPMJET3, QGSJET-II, EPOS and PYTHIA as shown in histograms. As shown in the figures, none of the models perfectly reproduce the data, while the data points are "bracketed" by the models. Among the models, DPMJET3 or PYTHIA would give too hard spectra in the high energy region. The shape of spectra of EPOS, QGSJET-II 

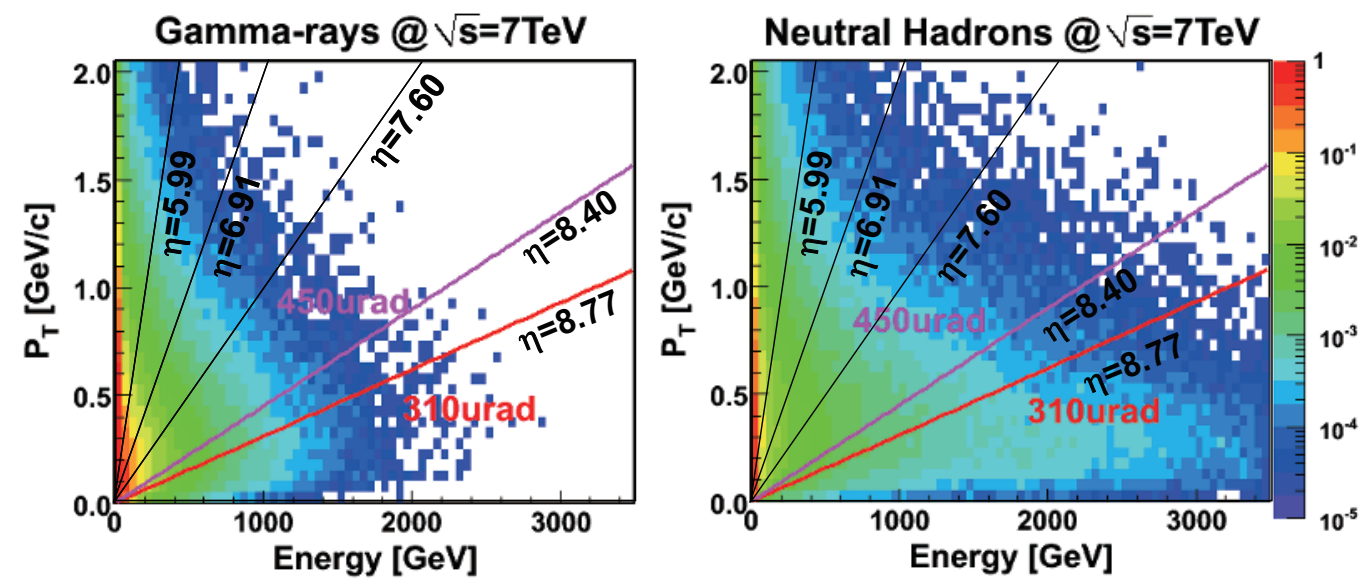

Figure 3. The energy vs $p_{T}$ for single gamma rays (left) and neutrons (right) from $7 \mathrm{TeV} p-p$ collisions simulated by DPMJET3. The lines shows the corresponding pseudorapidity $\eta=8.77,8.40,7.60,6.91$ and 5.99 (from bottom to top), respectively.
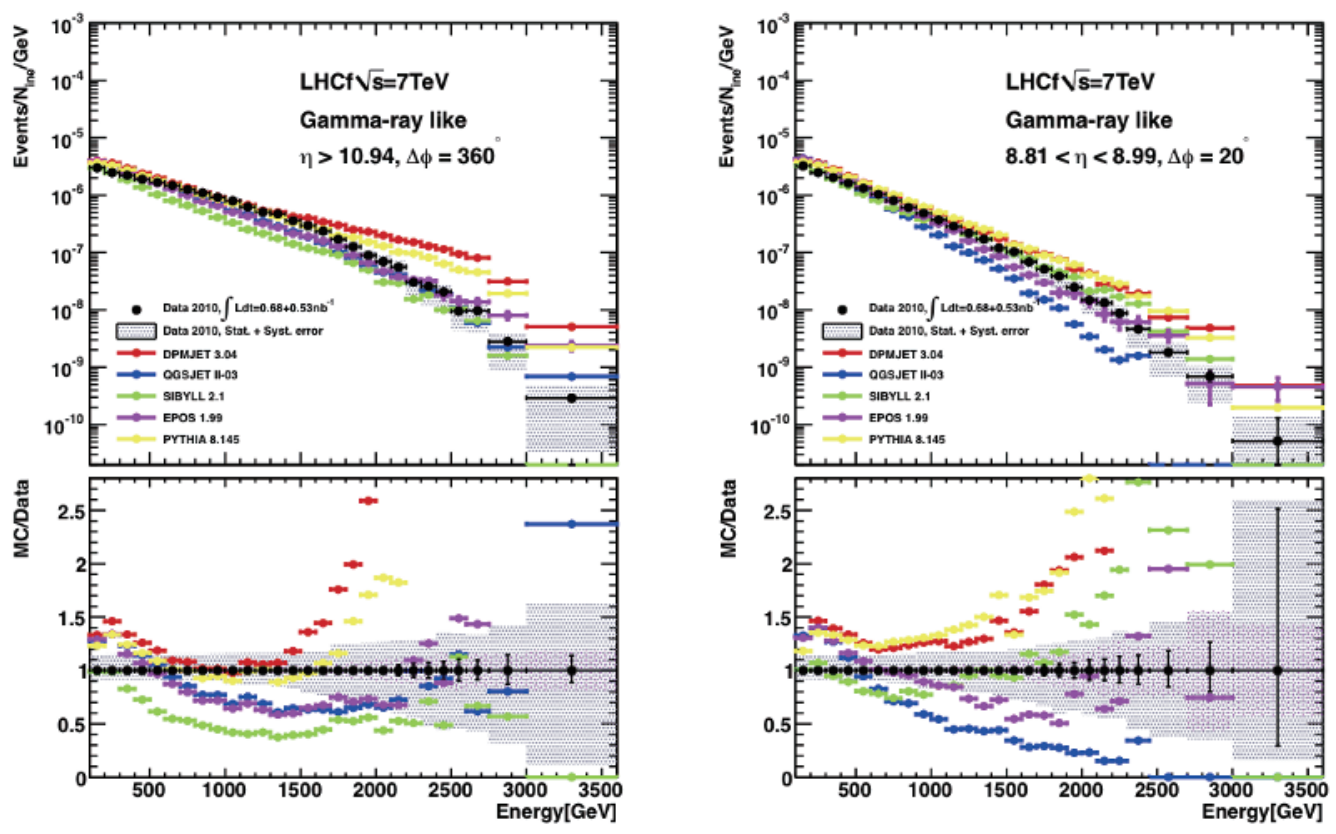

Figure 4. The black dots show the energy spectrum of single $\gamma$ sample in different rapidity ranges $\eta>10.94$ (left) and $8.81<\eta<8.99$ (right) at $7 \mathrm{TeV}$ p-p collisions [19]. Also shown the MC prediction by various hadron interaction models, SIBYLL 2.1 (green), EPOS 1.99 (purple), QGSJET II-03 (blue), PYTHIA 8.145 (yellow), and DPMJET3.04 (red). The top two and the bottom two panels show the event rate per collisions and the MC/Data ratios, respectively. 

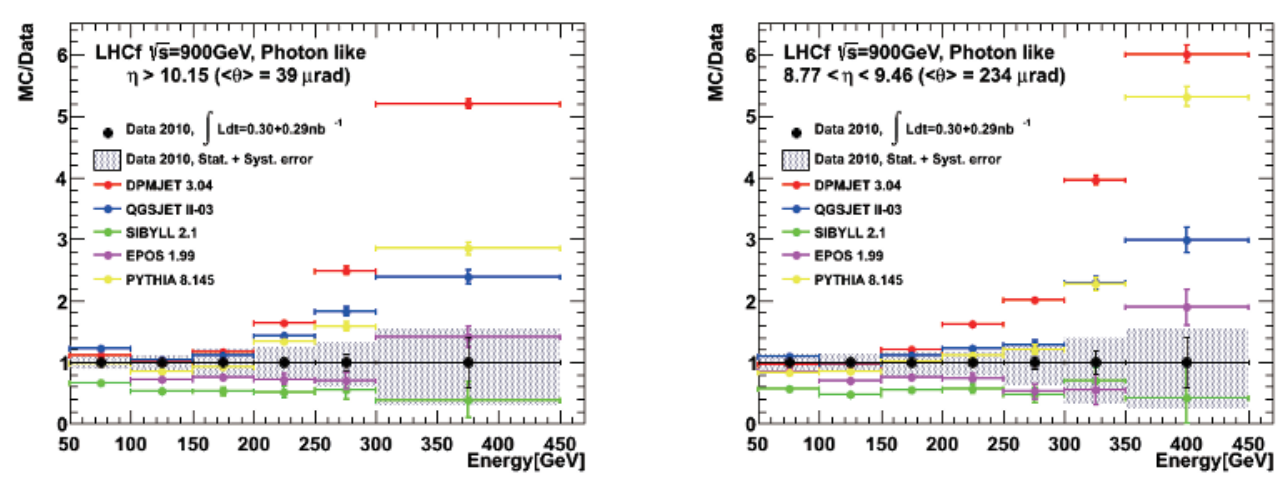

Figure 5. The black dots show the energy spectrum of single $\gamma$ sample in different rapidity ranges $\eta>10.15$ (left) and $8.77<\eta<9.46$ (right) at $0.9 \mathrm{TeV}$ p-p collisions [20]. Also shown the MC prediction by various hadron interaction models, SIBYLL 2.1 (green), EPOS 1.99 (purple), QGSJET II-03 (blue), PYTHIA 8.145 (yellow), and DPMJET3.04 (red). The top two and the bottom two panels show the differential cross section values and the MC/Data ratios, respectively.

and SYBILL give reasonable agreement with those of data within a experimental errors, while SYBILL gives lower yields than data. These features are commonly seen both in the $7 \mathrm{TeV}$ and $0.9 \mathrm{TeV}$ energy data. It might be a hint for future model improvement.

\section{$4 p_{T}$ spectra for the Type-I $\pi^{0}$ sample}

The $\pi^{0}$ sample provides a complemental information for the single $\gamma$ sample. Below the LHC energy, the UA7 data at $\mathrm{S} p \bar{p} \mathrm{~S}(\sqrt{s}=630 \mathrm{GeV})$ is available for the $p_{T}$ spectra of very forward $\pi^{0}$ production [21].

In the reconstruction of $\pi^{0}$, each $\gamma$ is reconstructed by one calorimenter ("Type-I" $\pi^{0}$ ). Limited by aperture of the beam pipe, $\pi^{0}$ 's with more than several hundred $\mathrm{GeV}$ can be reconstructed. On the other hand, two $\gamma$ 's from decay of a $\pi^{0}$ with more than $1.5 \mathrm{TeV}$ would be contained in a single calorimeter aperture ("Type-II" $\pi^{0}$ ). They could be also reconstructed their invariant masses thanks to fine granularity of position sensitive layers. Figure 6 shows invariant mass distributions for the Type-I and the Type-II $\pi^{0}$ samples. Mass resolution for Type-I and Type-II is 3.8 $\%$ and about $8 \%$, respectively. Still Type-II $\pi^{0}$ sample has resonablly good resolution, here we focus on Type-I $\pi^{0}$.

Figure 7 shows the $p_{T}$ spectra of Type-I $\pi^{0}$ 's reconstructed in the rapidity ranges $9.0<|y|<10.0$ at $7 \mathrm{TeV}$ $p-p$ collisions [22]. Also shown the $p_{T}$ spectrum predicted by the various hadron interaction models, DPMJET3.04, QGSJET II-03, SIBYLL 2.1, EPOS 1.99 and PYTHIA 8.145. Similar to the single $\gamma$ sample, none of the models perfectly agrees. However in the Type-I $\pi^{0}$ sample, EPOS gives pretty nice agreement with the data. This might be because Type-I $\pi^{0}$ s and the $\pi^{0}$ s for the origin of single $\gamma$ sample are not indentical. They originate in the different kienematical phase spaces (i.e. $P_{T}$ vs $E$ ).
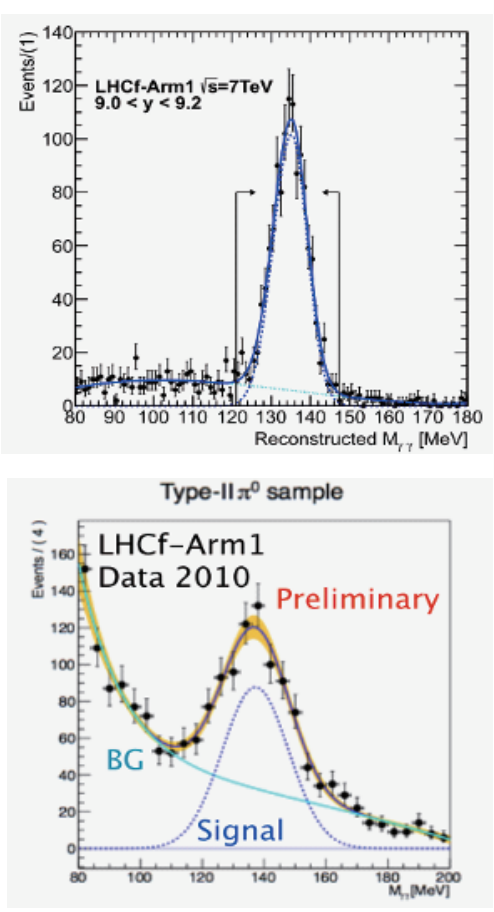

Figure 6. The invariant mass resolution for Type-I $\pi^{0}$ 's (top) and Type-II $\pi^{0}$ 's (bottom).

\subsection{Summary and Future prospects}

The LHCf data and also various LHC data including central rapidity regions have been already implemented into the cosmic ray intection models, for example, EPOS-LHC or QGSJET II-4. An impact of this improvement on air shower measurement has been discussed [23]. Difference of Xmax prediction at the $10^{20} \mathrm{eV}$ region becomes im- 

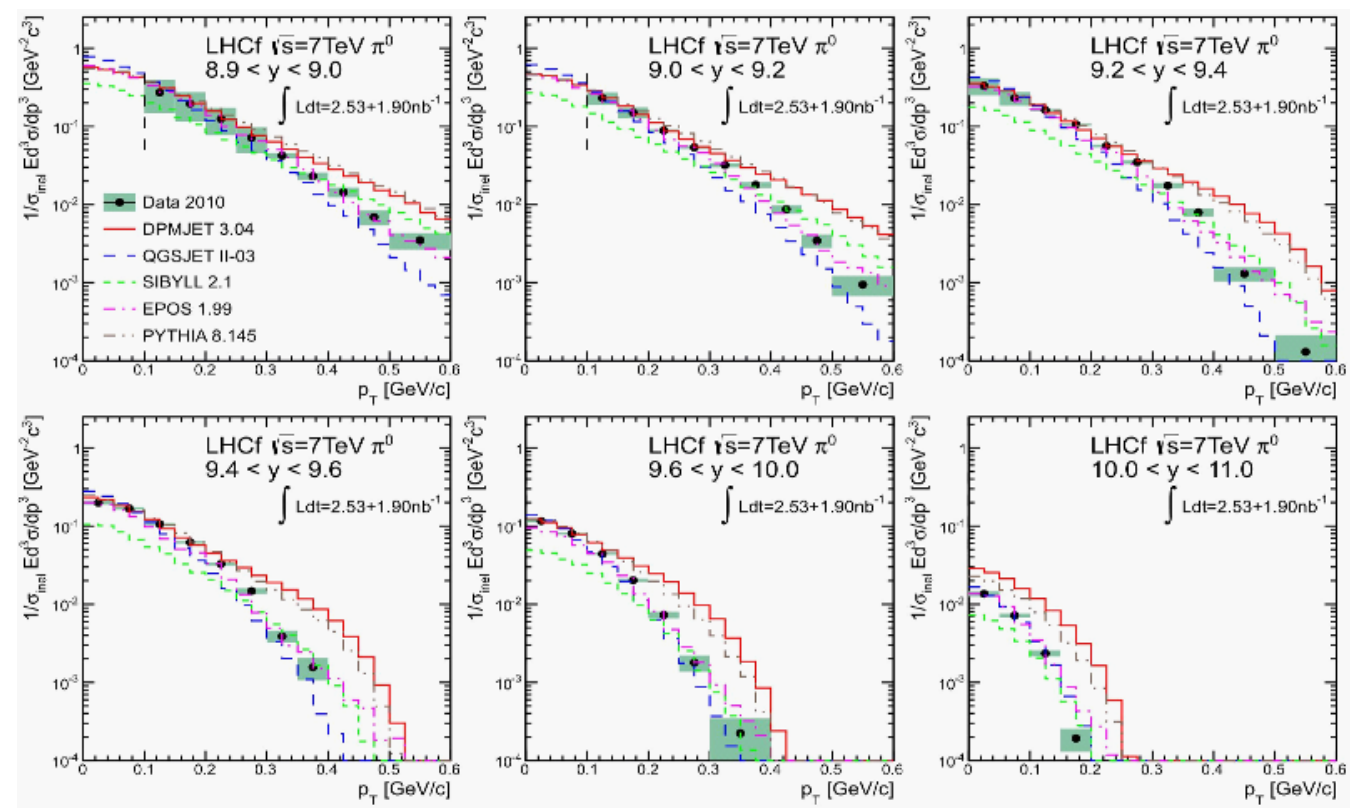

Figure 7. The $p_{T}$ spectra of Type-I $\pi^{0}$ 's reconstructed in six different rapidity ranges in $9.0<|y|<10.0$ at 7 TeV $p$ - $p$ collisions [22]. Also shown the $p_{T}$ spectrum predicted by the various hadron interaction models, DPMJET3.04 (red solid), QGSJET II-03 (blue long dashed), SIBYLL 2.1 (green short dashed), EPOS 1.99 (purple dotted dashed) and PYTHIA 8.145 (gray dotted).

proved and now has less energy-dependend. This clearly indicates benefit of LHC measurements to cosmic ray air shower experiments.

Further improvements will come from remaining issues; inelasticity, nuclear effects and energy dependence. The first issue can be probed by neutron measurement at very forward. Inelasticity of collisions controls how large fraction of hadronic cascade component in air showers would survive at deeper site of atmosphere. It could largely modify the prediction of muon density at the ground in the current air shower simulations. It might relate to the ground muon excess anomaly problem recently reported by PAO or KASCADE-GARNDE [1]. Currently the hadron shower analysis of LHCf data is under progress. It should give an interesting impact on this issue. Cold nuclear effect in the forward region is also an important issue. A new data on LHC $\mathrm{p}-\mathrm{Pb}$ lead collisions at $\sqrt{s_{N N}}=5 \mathrm{TeV}$ were obtained in Jan/Feb 2013. These data should be very interesting to verify nuclear effects based on the Glauber model picture used in cosmic ray interaction models. LHCf has been re-installed and successfully completed data taking.

Understanding energy evolution of cosmic ray interactions is also a big issue, when we extraporate knowledge at the LHC energy to the highest energy cosmic ray region i.e. $10^{20} \mathrm{eV}$. Early hint for the energy dependence could be seen in comparyson of $0.9 \mathrm{TeV}$ and $7 \mathrm{TeV}$ collisions. It will be further revealed by future $14 \mathrm{TeV}$ col- lisions data forseen at 2015. The LHCf expriment plans to revisit LHC at this opportunity. For this purpose, refabrication of the detector with rad-hard GSO scintillator is on-going [24]. Not only a new measurement at higher energy, but also new forward data at lower energy side is important to understand energy dependence. The accelerator layout for the interaction region at RHIC has similarity to the LHC machine. A new zero degree measurement at RHIC is under discussion. Here p-p collisions at $\sqrt{s}=0.5$ $\mathrm{TeV}$ is available. Also RHIC has rich experience for various nuclear beams. Possible light ion-light ion or protonlight ion collisions can probe cosmic ray interactions for proton-nitrogen or Iron-nitrogen. Moreover LHC has also such a capabiltiy for light ion collisions at UHECR energy. It is interesting opportunity for direct verification of cosmic ray interactions for air showers. These connections between LHC and cosmic rays will be a rich and unique program in future particle and cosmic ray physics.

\section{References}

[1] P. Abreu et al. [The Pierre-Auger collaboration], “ICRC2011, Beijing, Jul. 2011" arXiv:1107.4809

[2] Y. Tsunesada et al., [The Telscope Array collaboration], "ICRC2011, Beijing, Jul. 2011" arXiv:1111.2507

[3] W.D.Apel, et al., “ICRC2011, Beijing, Jul. 2011” , arXiv:1111.5436 
[4] C. Meurer et al., [AUGER collaboration], Astrophys. Space. Sci. Trans. 7, 183 (2011).

[5] G.Thomson, J. Phys. Conf. Ser, 47, 248 (2006).

[6] E.J. Ahn et al., Phys. Rev. D80, 094003 (2009).

[7] S. Ostapchenko, Phys. Lett. 636, 40 (2006).

[8] S. Roesler, R. Engel and J. Ranft, Proc. of 27th Int. Cosmic Ray Conf. 2002, 439.

[9] T. Pierog and K. Werner, Phys. Rev. Lett. 101, 171101 (2008).

[10] D. d'Enterria et al., Astropart. Phys. 35, 98 (2011).

[11] The CMS collaboration, JHEP 11, 148 (2011).

[12] The CMS collaboration, CMS PAS FWD-11-003.

[13] G.Antchev et al. [The TOTEM collaboration], Europhys. Lett. 98, 31002 (2012).

[14] H.Menjo et al. [LHCf collaboration], Astropart. Phys. 34, 513 (2011).

[15] K.Taki. [LHCf collaboration], JINST 7(2012), T01003.
[16] O.Adriani et al. [LHCf collaboration], JINST 3, S08006 (2008).

[17] T.Sako et al. [LHCf collaboration], Nucl. Instr. Meth. A578, 146 (2007).

[18] T.Mase et al. [LHCf collaboration], Nucl. Instr. Meth. A671, 129 (2012).

[19] O.Adriani et al. [LHCf collaboration], Phys. Lett. B703, 128, (2011).

[20] O.Adriani et al. [LHCf collaboration], Phys. Lett. B715, 298 (2012).

[21] E. Paré et al. [UA7 collaboration], Phys. Lett. 242, 531 (1990).

[22] O.Adriani et al. [LHCf collaboration], Phys. Rev. D86, 092001 (2012).

[23] T.Pierog, "LHC data and extensive air shower", ISVHECRI2012, 10-15 Aug., 2012. Berlin.

[24] K.Kawade et al., JINST 6 T09004 (2011). 